\title{
Mutual Contextualization in Tripartite Graphs of Folksonomies
}

\author{
Ching-man Au Yeung, Nicholas Gibbins, and Nigel Shadbolt \\ Intelligence, Agents and Multimedia Group (IAM), \\ School of Electronics and Computer Science, \\ University of Southampton, \\ Southampton SO17 1BJ, UK \\ \{cmay06r,nmg,nrs\}@ecs.soton.ac.uk
}

\begin{abstract}
The use of tags to describe Web resources in a collaborative manner has experienced rising popularity among Web users in recent years. The product of such activity is given the name folksonomy, which can be considered as a scheme of organizing information in the users' own way. This research work attempts to analyze tripartite graphs - graphs involving users, tags and resources - of folksonomies and discuss how these elements acquire their semantics through their associations with other elements, a process we call mutual contextualization. By studying such process, we try to identify solutions to problems such as tag disambiguation, retrieving documents of similar topics and discovering communities of users. This paper describes the basis of the research work, mentions work done so far and outlines future plans.
\end{abstract}

\section{Introduction}

The use of freely-chosen words or phrases called tags to classify Web resources has experienced rising popularity among Web users in recent years. Through the use of tags, Web users come to share and organize their favourite Web resources in different collaborative tagging systems, such as del.icio.us 1 and Flickn 2 . The result of such social and collaborative tagging is given the name folksonomy [1].

Collaborative tagging and folksonomy possess a number of advantages which account for its popularity [4. These include its simplicity as well as the freedom enjoyed by the users to choose their own tags. Folksonomies have also been considered to be a possible solution to construct ontologies which can be used in the Semantic Web [7. However, some limitations and shortcomings, such as the problem of ambiguous meanings of tags and the existence of synonyms, also affect its effectiveness to organize resources on the Web [4].

In this research, we focus on analysis of tripartite graphs of folksonomies, graphs which involves the three basic elements of collaborative tagging, namely users, tags and resources. We investigate how these elements come to acquire

${ }^{1}$ http://del.icio.us/

${ }^{2}$ http://www.flickr.com/

K. Aberer et al. (Eds.): ISWC/ASWC 2007, LNCS 4825, pp. 966 970. 2007.

(C) Springer-Verlag Berlin Heidelberg 2007 
their own semantics through their associations with other elements in the graphs, a process which we call mutual contextualization. The study aims to acquire a more thorough understanding of the characteristics of folksonomies, and to devise mechanisms that allow applications to better utilize the power of folksonomies. Methods of network analysis and community discovery will be employed to reveal the semantics hidden in the seemingly chaotic folksonomy data.

\section{Mutual Contextualization in Folksonomies}

The power of folksonomies lies in the interrelations between the three elements. A tag is only a symbol if it is not assigned to some Web resources. A tag is also ambiguous without a user's own interpretation of its meaning. Similarly, a user, though identified by its username, is characterized by the tags it uses and the resources it tags. Finally, a document is given semantics because tags act as a form of annotation. Hence, it is obvious that each of these elements in a folksonomy would be meaningless, or at least ambiguous in meaning, if they are considered independently. In other words, the semantics of one element depends on the context given by the other two, or all, elements that are related to it.

To further understand this kind of mutual contextualization, we examine each of the three elements in a folksonomy in details. For more specific discussions, we assume that the Web resources involved are all Web documents. In addition, we define the data in a social tagging system, a folksonomy, as follows.

Definition 1. A folksonomy $F$ is a tuple $F=(U, T, D, A)$, where $U$ is a set of users, $T$ is a set of tags, $D$ is a set of Web documents, and $A \subseteq U \times T \times D$ is a set of annotations.

As we have mentioned earlier, the three elements forming the tripartite graph of a social tagging system are users, tags and documents (resources). The tripartite graph can be reduced into a bipartite graph if, for example, we focus on a particular tag and extract only the users and documents that are affiliated to it. Since there are three types of elements, there can be three different types of bipartite graphs. This is similar to those introduced by Mika [7], but we distinguish our method by only concentrating on one instance of an element instead of all the instances of the same element.

\section{$2.1 \quad$ Users}

By focusing on a single user $u$, we obtain a bipartite graph $T D_{u}$ defined as follows:

$$
T D_{u}=\left\langle T \times D, E_{t d}\right\rangle, E_{t d}=\{(t, d) \mid(u, t, d) \in A\}
$$

In other words, an edge exists between a tag and a document if the user has assigned the tag to the document. The graph can be represented in matrix form, which we denote as $\mathbf{X}=\left\{x_{i j}\right\}, x_{i j}=1$ if there is an edge connecting $t_{i}$ and $d_{j}$. The bipartite graph represented by the matrix can be folded into two one-mode networks [7]. We denote one of them as $\mathbf{P}=\mathbf{X X}^{\prime}$, and another as $\mathbf{R}=\mathbf{X}^{\prime} \mathbf{X}$. 
$\mathbf{P}$ represents a kind of semantic network which shows the associations between different tags. It should be note that this is unlike the light-weight ontology mentioned in [7, as it only involves tags used by a single user. In other words, this is the personal vocabulary used by the particular user, which can be called a personomy [5].

The matrix $\mathbf{R}$ represents the personal repository of the user. Links between documents are weighted by the number of tags that have been assigned to both documents. Thus, documents having higher weights on the links between them are those that are considered by the particular user as more related.

By studying and comparing the documents and tags associated with different users, we can have a better understanding of the vocabularies as well as the interests or expertise [6] of different users. This will lead to more efficient and effective ways for matching user interests and discovering community of users.

\subsection{Tags}

By using a similar method as described above, we can obtain a bipartite graph $U D_{t}$ regarding to a particular tag $t$ :

$$
U D_{t}=\left\langle U \times D, E_{u d}\right\rangle, E_{u d}=\{(u, d) \mid(u, t, d) \in A\}
$$

In words, an edge exists between a user and a document if the user has assigned the tag $t$ to the document. The graph can once again be represented in matrix form, which we denote as $\mathbf{Y}=\left\{y_{i j}\right\}, y_{i j}=1$ if there is an edge connecting $u_{i}$ and $d_{j}$. This bipartite graph can be folded into two one-mode networks, which we denote as $\mathbf{S}=\mathbf{Y} \mathbf{Y}^{\prime}$, and $\mathbf{C}=\mathbf{Y}^{\prime} \mathbf{Y}$.

The matrix $\mathbf{S}$ shows the affiliation between the users who have used the tag $t$, weighted by the number of documents to which they have both assigned the tag. Since a tag can be used to represent different concepts (such as $s f$ for San Francisco or Science Fiction), and a document provides the necessary content to identify the contextual meaning of the tag, this network is likely to connect users who use the tag for the same meaning.

C can be considered as another angle of viewing the issue of polysemous or homonymous tags. Thus, with the edges weighted by the number of users who have assigned tag $t$ to both documents, this network is likely to connect documents which are related to the same sense of the given tag.

The existence of ambiguous tags and synonymous tags is a major problem in folksonomies. These tags affect the precision of describing resources as well as that of retrieval of relevant documents. By examining the documents and users associated with a tag, we are actually trying to interpret the meaning of a tag by placing it in the right context. Thus, this will probably provides a possible way to understanding the semantics of tags in a folksonomy.

\subsection{Documents}

Finally, a bipartite graph $U T_{d}$ can also be obtained by considering a particular document $d$. The graph is defined as follows:

$$
U T_{d}=\left\langle U \times T, E_{u t}\right\rangle, E_{u t}=\{(u, t) \mid(u, t, d) \in A\}
$$


In words, an edge exists between a user and a tag if the user has assigned the tag to the document $d$. The graph can be represented in matrix form, which we denote as $\mathbf{Z}=\left\{z_{i j}\right\}, z_{i j}=1$ if there is an edge connecting $u_{i}$ and $t_{j}$. Like in the cases of a single user and a single tag, this bipartite graph can be folded into two one-mode networks, which we denote as $\mathbf{M}=\mathbf{Z} \mathbf{Z}^{\prime}$, and $\mathbf{V}=\mathbf{Y}^{\prime} \mathbf{Y}$.

The matrix $\mathbf{M}$ represent a network in which users are connected based on the documents commonly tagged by them. Since a document may provide more than one kind of information, and users do not interpret the content from a single perspective, the tags assigned by different users will be different, although tags related to the main theme of the document are likely to be used by most users. Hence, users linked to each other by edges of higher weights in this network are more likely to share a common perspective, or are more likely to concern a particular piece of information provided by the document.

On the other hand, the matrix $\mathbf{V}$ represents a network in which tags are connected and weighted by the number of users who have assigned them to the document. Hence, the network is likely to reveal the different perspective of the users from which they interpret the content of the document.

The bipartite graph obtained by focusing on a single document gives us information on how different users interpret the content of the document, and which aspects in the document do they focus on. A better understanding of these issues will surely benefit applications such as Web page recommender systems.

From the above discussions, we can see that different relations between the users, the tags and the documents in a folksonomy will affect how a single user, tag or document is interpreted in the system. Each of these elements provide an appropriate context such that the semantics of the elements can be understood without ambiguity.

\section{Research Progress and Future Plan}

The current stage of this research work focuses on bipartite graphs obtained by examining a single tag. As noted by several other authors 148, there are actually quite a lot of ambiguous tags in existing folksonomies. One of the objectives of studying the bipartite graphs is to discover effective methods for tag meaning disambiguation. To illustrate the approach consider the following example. The tag $s f$ is observed to be used to represent two distinctive concepts, namely "science fiction" and "San Francisco." The result shows that users and documents which are associated with the same meanings of the tag tend to be clustered with each other. This shows that it is possible to disambiguate tags by studying the bipartite graphs. We are currently carrying out research on how different community discovering techniques (e.g. [23]) can be employed to develop an algorithm for tag disambiguation.

The future plan of this research involves the following steps. Firstly, after the study of tag disambiguation, we will move on to study bipartite graphs obtained by examining a single document or a single user. We will investigate how similar techniques of network analysis can be applied to these graphs to discover useful 
information regarding interests and expertise of users, communities of users with shared interests, and clusters of documents with similar topics. In the end we hope to develop a unified theory of the process of mutual contextualization in folksonomies, which gives a thorough picture of the semantics of the elements involved. Finally, we will investigate how these results can be applied to applications such as social network analysis, Web page recommender systems and construction of ontologies.

\section{Conclusion}

This paper describes a research work on the analysis of tripartite graphs of folksonomies. In particular, we study the process of mutual contextualization between the three basic elements in a folksonomy, in the hope of understanding the semantics of these elements. Promising results have been obtained in preliminary studies of disambiguation of tags, and plans for future works have been drawn. We believe this work will contribute to a better understanding of folksonomies and benefit different applications on the Web.

\section{References}

1. Adam, M.: Folksonomies - cooperative classification and communication through shared metadata (2004), http://www.adammathes.com/academic/computermediated-communication/folksonomies.html

2. Clauset, A.: Finding local community structure in networks. Physical Review E 72(026132) (2005)

3. Girvan, M., Newman, M.E.J.: Community structure in social and biological networks. Proc. Natl. Acad. Sci. USA 99(7821) (2002)

4. Golder, S., Huberman, B.A.: Usage patterns of collaborative tagging systems. Journal of Information Science 32(2), 198-208 (2006)

5. Hotho, A., Jäschke, R., Schmitz, C., Stumme, G.: Information retrieval in folksonomies: Search and ranking. In: Sure, Y., Domingue, J. (eds.) ESWC 2006. LNCS, vol. 4011, pp. 411-426. Springer, Heidelberg (2006)

6. John, A., Seligmann, D.: Collaborative tagging and expertise in the en- terprise. In: WWW 2006: Proceedings of the 15th international conference on World Wide Web, ACM Press, New York (2006)

7. Mika, P.: Ontologies are us: A unified model of social networks and semantics. In: International Semantic Web Conference, pp. 522-536 (2005)

8. Wu, X., Zhang, L., Yu, Y.: Exploring social annotations for the semantic web. In: WWW 2006. Proceedings of the 15th international conference on World Wide Web, pp. 417-426. ACM Press, New York (2006) 